\title{
The Thiamine Analogue and Advanced Glycation Endproducts Crosslink Breaker ALT-711 does not Interfere with Transketolase Activity
}

\author{
Martina Krautwald ${ }^{1}$, Philippa Maxwell ${ }^{1}$, Grant Stuchbury ${ }^{2}$, Joe Holtum ${ }^{3}$, James Burnell ${ }^{1}$ and \\ Gerald Münch*,1 \\ ${ }^{I}$ Department of Pharmacology, School of Medicine, University of Western Sydney, Campbelltown, Australia \\ ${ }^{2}$ Department of Biochemistry and Molecular Biochemistry, School of Pharmacy and Molecular Science, James Cook \\ University, Townsville, Australia \\ ${ }^{3}$ Department of Tropical Plant Sciences, School of Tropical Biology, James Cook University, Townsville, Australia
}

\begin{abstract}
The enzyme transketolase (sedoheptulose-7-phosphate:D-glyceraldehyde-3-phosphate glycolaldehydetransferase, EC 2.2.1.1) is involved in the pentose phosphate pathway (PPP) and catalyses the transfer of a 2-carbon fragment from a 5-carbon keto sugar (xylulose-5-P) to a 5-carbon aldo sugar (ribose-5-P) to form a 7-carbon keto sugar (sedoheptulose-7-P) and a 3-carbon aldo sugar (glyceraldehyde-3-P). Transketolase requires thiamine pyrophosphate as a co-factor. Advanced glycation endproducts (AGEs) are implicated in the complications of diabetes and aging, primarily via adventitious and crosslinking of tissue proteins. ALT-711 is an AGE crosslink breaker and has been tested as an intervention therapy in established complications of diabetes. It has been noticed that it has a similar structure to that of thiamine and it was hypothesized that it might inhibit transketolase by replacing the active co-factor rendering the enzyme inactive. In this study, we have established a novel microtiter plate format transketolase assay which determines the concentration of NADH by measuring its fluorescence. Using this assay, it was found that ALT-711 does not inhibit the activity of transketolase up to concentration of $5 \mathrm{mM}$. We conclude that ALT-711 does not interfere with transketolase activity at clinically relevant concentrations.
\end{abstract}

Keywords: ALT-711, glycation, crosslink breaker, transketolase, co-factor, thiamine pyrophosphate, carbonyl stress.

\section{INTRODUCTION}

The accumulation of advanced glycation end products (AGEs) is responsible for many complications of diabetes [1]. Many of the effects of AGEs are due to their ability to cause crosslinking of proteins. Collagen is a primary example of a protein that commonly becomes crosslinked, which results in stiffening of the connective tissue in arteries and the skin [2]. ALT-711 has been proposed to break AGE crosslinks and has proved to be successful as an intervention therapy in established complications in diabetes in a variety of animal models [3-6]. Whilst ALT-711 has many advantageous properties, it has been noticed, that it and its major metabolite ALT-1235, have similar structures to that of thiamine (Fig. 1). Thiamine is a precursor of thiamine pyrophosphate (TPP). TPP is an essential cofactor for enzymes such as pyruvate dehydrogenase (E1 subunit), $\alpha$ ketoglutarate dehydrogenase $(\alpha-\mathrm{KGDH})$, branched chain $\alpha$ keto acid dehydrogenase and transketolase (TK) [7]. A decreased activity of these enzymes due to TPP deficiency results in serious disorders such as Beriberi and WernickeKorsakoff syndrome [8-10]. The enzyme TK (sedoheptulose-7-phosphate:D-glyceraldehyde-3-phosphate glycolaldehydetransferase, EC 2.2.1.1) is involved in the pentose phos-

*Address correspondence to this author at the Department of Pharmacology, School of Medicine, University of Western Sydney, Locked Bag 1797, Penrith South DC 1797, NSW, Australia; Tel: +61 29852 4718; E-mail: g.muench@uws.edu.au phate pathway (PPP) and catalyses the transfer of a 2-carbon fragment from a 5-carbon keto sugar (xylulose-5-P) to a 5carbon aldo sugar (ribose-5-P) to form a 7-carbon keto sugar (sedoheptulose-7-P) and a 3-carbon aldo sugar (glyceraldehyde-3-P) $[11,12]$. TK, the rate limiting enzyme in the nonoxidative part of the PPP, plays a crucial role in nucleic acid ribose synthesis utilising glucose carbons in tumour cells [13]. TK has been suggested as a promising drug target in the treatment of cancer [14], based on the fact that thiamine analogues can replace the non-covalently bound co-factor TPP, rendering the enzyme inactive [15]. Should ALT-711 compete with TPP for TK, there could be serious side-effects associated with the drug, particularly after long-term exposure. The importance of active TK is particularly important in diabetes, since the accumulation of triosephosphates and the increased formation of the potent glycating agent methylglyoxal and methylglyoxal-derived AGEs in intracellular hyperglycaemia are implicated in the development of diabetic complications [16]. Thiamine supplementation increases the activity of TK, decreases the concentration of the triosephosphate pool, the concentration and metabolic flux of the formation of methylglyoxal, and increases the concentration of total sedoheptulose-7-phosphate and ribose-5phosphate [17]. In contrast, intracellular methylglyoxal levels are increased in animals on a thiamine-reduced diet [18].

It was hypothesized that ALT-711 could actually increase AGE levels if it would inhibit TK. Therefore, the aim of this study was to determine if ALT-711 is able to inhibit TK activity in vitro. 

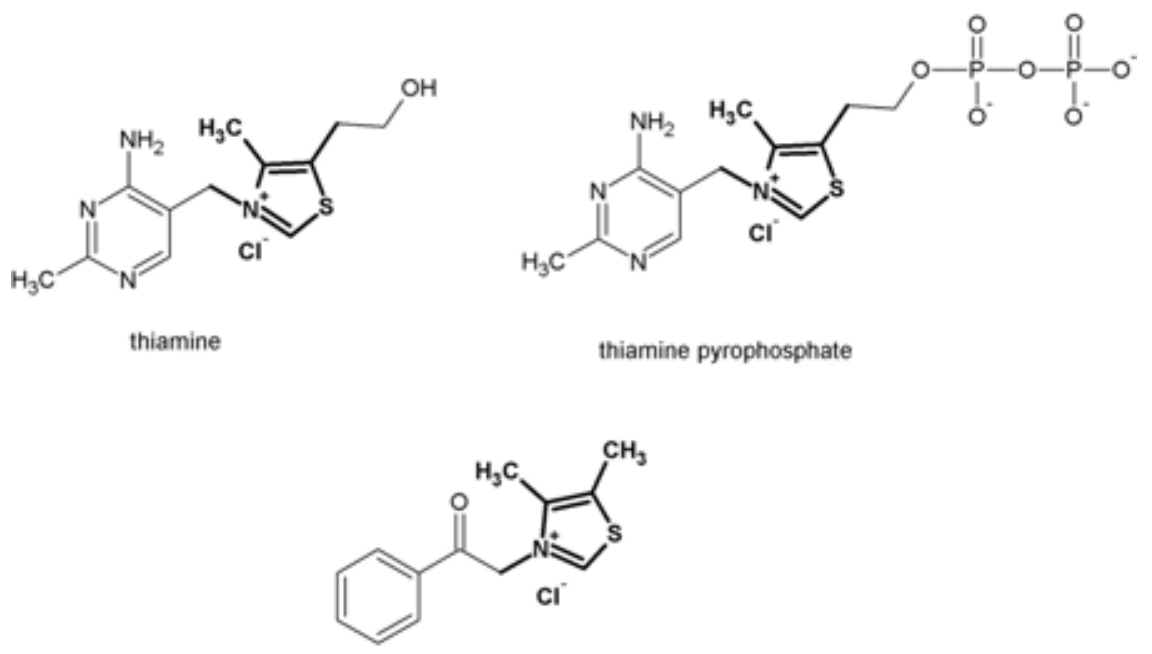

ALT-711

Fig. (1). Structure of thiamine, thiamine pyrophosphate and the crosslink breaker ALT-711.

\section{MATERIALS AND METHODOLOGY}

\section{Materials}

Triosephosphate isomerase (TPI) and $\alpha$-glycerophosphate dehydrogenase $(\mathrm{GDH})$ were from Boehringer Mannheim. Fructose-1,6-bisphosphate (F-1,6-P), NADH, $\mathrm{MgCl}_{2}$, TRIS, $\mathrm{HCl}$, Ribose-5-phosphate (R-5-P) were purchased from Sigma. $\beta$-mercaptoethanol was from Merck and ALT-711 was donated by Synvista Therapeutics.

\section{Methodology}

\section{Protein Extraction from Liver}

$102 \mathrm{~g}$ of frozen chicken liver were homogenized with a handheld blender in $510 \mathrm{~mL}$ of a TRIS-HCl buffer $(20 \mathrm{mM}$, $\mathrm{pH}$ 7.4) containing $2 \mathrm{mM} \beta$-mercaptoethanol and $1 \mathrm{mM}$ EDTA. After centrifugation at $10,000 \mathrm{~g}$ for $10 \mathrm{~min}$ the supernatant was removed and kept at $4{ }^{\circ} \mathrm{C}$ for subsequent enzymatic reactions.

\section{Transketolase Assay}

The activity of TK in chicken liver extract was determined following the protocol of Chamberlain and Thornalley with modifications $[17,19]$. Briefly, an aliquot $(200 \mu \mathrm{L})$ of substrate cocktail (14.8 mM R-5-P, $253 \mathrm{mM}$ NADH, 185 $\mathrm{U} / \mathrm{mL}$ TPI, and $70 \mathrm{~mL}$ of $21.5 \mathrm{U} / \mathrm{mL} \mathrm{GDH}$ in $250 \mathrm{mM}$ Tris/HCl buffer, $\mathrm{pH} 7.8$ ) was added to the wells of a $96-$-well microtiter plate and up to $20 \mu \mathrm{L}$ of the chicken liver extract. The absorbance at $340 \mathrm{~nm}$ was monitored at 10 min intervals for $2 \mathrm{~h}$ and the rate of decrease in fluorescence between 20 to $110 \mathrm{~min}$ was used to deduce the rate of oxidation of NADH in the GDH catalyzed reaction, which is rate limited by the TK catalyzed conversion of R-5-P and xylulose-5-phosphate to sedoheptulose-7-phosphate and GA3P under these conditions. For inhibition experiments, $20 \mu \mathrm{L}$ of liver homogenate were preincubated with different concentrations of ALT-711 $(0.5 \mathrm{mM}-10 \mathrm{mM})$ before the reaction was started. A negative control that contained no R-5-P, and a positive control, which contained no fluorescence was measured as previously described, with measurements at 10 min intervals. Reaction rates for the TK assays containing ALT-711 were calculated based on a standard curve of NADH fluorescence. The same reaction was also performed using $1 \mathrm{~mL}$ cuvettes, with the volumes adjusted proportionally to a final volume of $500 \mu \mathrm{L}$. The absorbance was then measured at $340 \mathrm{~nm}$ every $10 \mathrm{~min}$ for $2 \mathrm{~h}$.

\section{Data Analysis}

Experiments were performed in triplicates and data are presented as mean \pm SEM. Graphs were constructed and analyzed with Graphpad Prism 5 software (ISI Software San Diego, CA, USA).

\section{RESULTS}

\section{Establishment of the TK assay in Microtiter Plate For- mat with Fluorescence Detection}

The activity of TK was measured using a linked enzyme assay with NADH as the "reporter" product. The conventional method of TK activity assay uses ribose-5-phosphate and xylulose-5-phosphate as substrates [20]. However, we had to establish a new method of the TK assay since xylulose-5-phosphate is no longer commercially available. However, ribose-5-phosphate can be used as the sole substrate since the second substrate, xylulose-5-phosphate, can be produced from ribose-5-phosphate in situ through the action of ribulose-5-phosphate isomerase and ribulose-5-phosphate epimerase (Fig. 2). To measure TK activity, glyceraldehyde3-phosphate produced by TK is converted to dihydroxyacetonephosphate by triosephosphate isomerase, then reduced to $\alpha$-glycerophosphate by glycerophosphate dehydrogenase oxidizing one NADH molecule to $\mathrm{NAD}^{+}$in the process, which can be measured spectrophotometrically (Fig. 2). $\mathrm{NADH}$ (but not $\mathrm{NAD}^{+}$) has an absorbance maximum at 340 $\mathrm{nm}$ and is usually detected by UV absorbance spectrophotometry. However, it can be detected even much more sensitively by fluorescence spectrometry with excitation/emission wavelengths set at 360/460 $\mathrm{nm}$, respectively [21]. As the NADH decreases proportial to glyceraldehyde-3-phosphate, the activity of TK can be determined by monitoring the loss of NADH fluorescence over time [22]. 


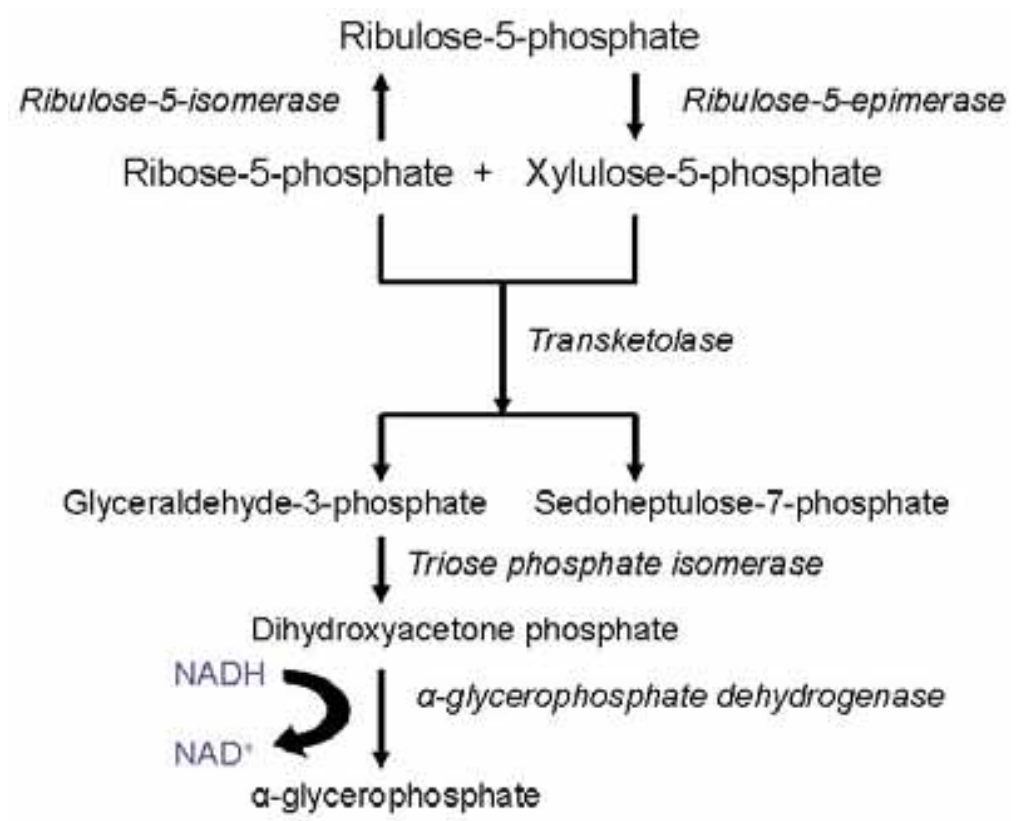

Fig. (2). Principle of the TK assay.

The rate of oxidation of NADH in the GDH catalyzed reaction controlled by the TK catalyzed conversion of ribose-5-phosphate and xylulose-5-phosphate to sedoheptulose-7-phosphate and GA3P under these conditions. Xylulose-5-phosphate is generated in situ from ribose-5phoshpate.

In a first set of experiments, a fluorescence based microtiter plate assay was established, which allows to use reduced reaction volumes and decreases the substantial costs for the reagents. To determine the reaction rate accurately, it was particularly important to determine the linear phase of the reaction and proves a dose- and substrate dependent TK activity. An enzyme-dependent increase in TK activity could be clearly detected since the addition of increasing volumes of liver extract (= increase in the concentration of active TK) lead to a steady increase in the reaction rate (Fig. 3). As expected, the highest activity was obtained with the highest enzyme concentration (present in $20 \mu \mathrm{L}$ liver extract), and therefore $20 \mu \mathrm{l}$ liver extract was chosen for all further experiments (Fig. 3). The time points at 10 minutes often showed a spike in fluorescence (most likely caused by a mixing artefact), and therefore the reaction rates were calculated between 20 and 80 minutes.

\section{TK Activity in the Presence of ALT-711}

A potential inhibitory effect of ALT-711 on TK activity was determined after preincubating the liver extract with various concentrations of the drug (to allow replacement of TPP by ALT-711) for 10 minutes before starting the reaction. The reaction rates of samples containing ALT-711 are very similar to those of the positive control containing only liver extract. Furthermore, it was observed that ALT-711 with concentrations up to $5 \mathrm{mM}$ does not inhibit the activity of TK (Fig. 4). Using a standard curve of fluorescence unit

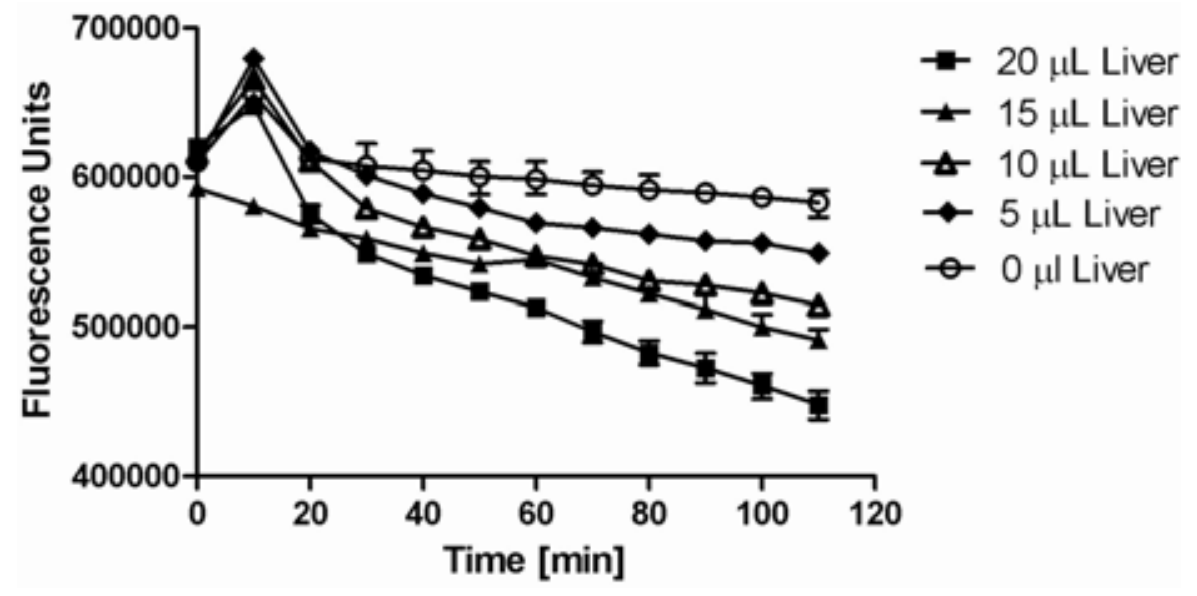

Fig. (3). TK assay with fluorescence detection.

TK activity was measured using a linked enzyme assay with NADH as the "reporter" product, expressed as fluorescence units (FU). The reaction rate was calculated in linear phase of the reaction ranging from $20 \mathrm{~min}$ up to $80 \mathrm{~min}$. The highest activity was obtained with the highest enzyme concentration (present in $20 \mu \mathrm{L}$ liver extract), and this enzyme concentration was chosen for all further experiments. 


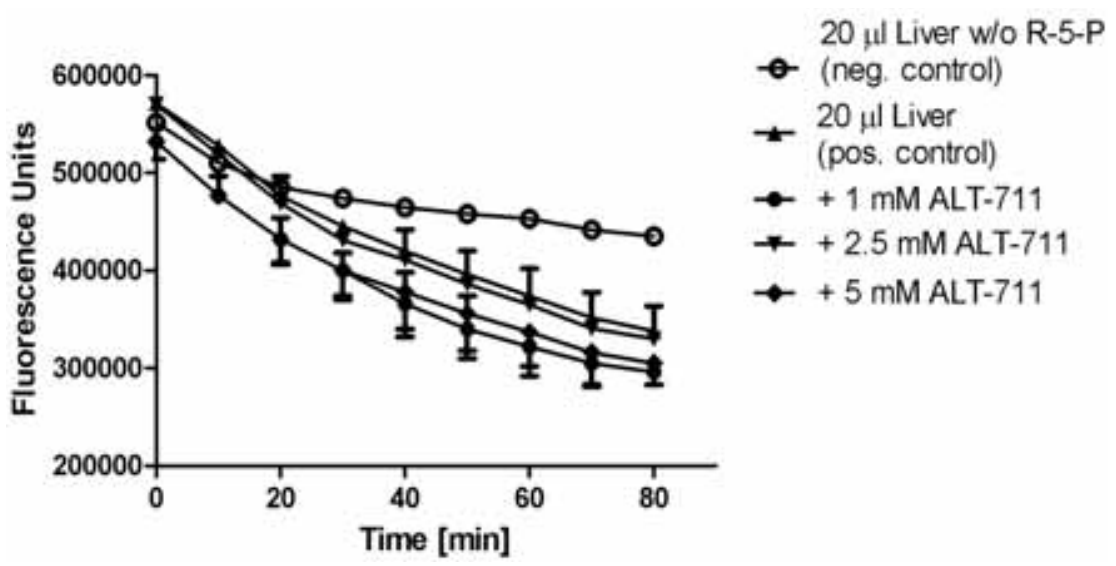

Fig. (4). Activity of TK in the presence of ALT-711.

A potential inhibitory effect of ALT-711on TK activity was determined after preincubating the liver extract with various concentrations of the drug. [NADH] as the assay readout was determined by fluorescence and is expressed as fluorescence units (FU). The reaction rates of samples containing ALT-711 are very similar to those of the positive control containing only liver extract, suggesting that ALT-711 does not inhibit TK activity up to a concentration of $5 \mathrm{mM}$.

vs $[\mathrm{NADH}]$, the reaction rates of TK alone, and in the presence of ALT-711 were calculated (Table 1). The negative control, with the reaction mix without the substrate ribose-5phosphate, had a reaction rate of $0.65 \mu \mathrm{M} \mathrm{NADH} / \mathrm{min}$, whereas $2 \mu \mathrm{M} \mathrm{NADH} / \mathrm{min}$ were used by TK present in 20 $\mu \mathrm{L}$ of liver extract in the presence of the substrate. ALT-711 did not inhibit TK activity up to a concentration of $5 \mathrm{mM}$, and demonstrated a (non-significant) slight increase in activity compared to the control, as the rates of $\mathrm{NAD}^{+}$production vary between $2.2 \mu \mathrm{M} /$ minute and $2.4 \mu \mathrm{M}$ /minute. When the experiments were repeated in $0.5 \mathrm{~mL}$ cuvettes detecting NADH by UV absorbance, no difference between the ALT711 and control samples were recorded. The only difference was that the drop in NADH signal in the negative control was not observed (data not shown).

Table 1. Effect of ALT-711 on TK Activity

\begin{tabular}{|c|c|}
\hline [ALT-711] $(\mathbf{m M})$ & Reaction Rate [NADH] $(\boldsymbol{\mu M} / \mathbf{m i n})$ \\
\hline \hline 0 & 2.0 \\
\hline 1.0 & 2.4 \\
\hline 2.5 & 2.3 \\
\hline 5.0 & 2.2 \\
\hline
\end{tabular}

\section{DISCUSSION}

In vitro and in vivo investigations suggest that ALT-711 (Alagebrium) is a breaker of AGE-derived cross-linking [23]. Specifically, oral administration of ALT-711 to diabetic rats results in a dose-dependent reduction of IgG-RBC crosslinks. Furthermore, rat, dog and non-human primate animal models have each demonstrated the beneficial effects of ALT-711 in reversing some of the cardiovascular complications resulting from aging, hypertension, and diabetes [1, $4,24]$. Since ALT-711 exhibits a close structural homology to thiamine (Vitamin B1) we hypothesized that it might be a possible inhibitor for thiamine pyrophosphate containing enzymes such as TK. In a further study we have shown that
ALT-711 acts as a low-affinity competitive inhibitor of thiamine pyrophosphokinase (Krautwald and Münch, unpublished observation). In this study we show that ALT-711 - at least with the short pre-incubation time of 10 minutes - does not inhibit the activity of TK up to a concentration of $5 \mathrm{mM}$. Although pharmakokinetic data of ALT-711 in humans have not been published, ALT-711 was given in doses up to 420 $\mathrm{mg}$ /day [25]. If one compares this dose to the recommended daily intake of thiamine in adults $(1.2 \mathrm{mg} /$ day for males, 1.1 $\mathrm{mg} /$ day for females) [26], it is unlikely that ALT-711 concentrations exceed low micromolar concentrations in plasma or tissues. Therefore, we suggest that ALT-711 does not interfere with transketolase activity at clinically relevant concentrations and is therefore unlikely to lead to increased methylglyoxal levels via this mode of action.

\section{CONCLUSION}

Although ALT-711 exhibits a close structural homology to thiamine (Vitamin B1), it does not replace the transketolase co-factor thiamine pyrophosphate. An inhibition in TK activity would increase the accumulation of triosephosphates and therefore increase the concentration of methylglyoxal and methylglyoxal-derived AGEs which are implicated in the development of diabetic complications, and therefore counteract the anti-AGE properties of the drug. However, our data show that ALT-711 is unlikely to inhibit transketolase activity and cause any serious side effects via this mechanism at clinically relevant concentrations.

\section{ACKNOWLEDGEMENT}

This work was supported by the Interdisciplinary Centre of Clinical Research (IZKF) at the Faculty of Medicine of the University of Leipzig (01KS9504, Project N1), James Cook University (special topics teaching fund) and Synvista Therapeutics. Many thanks go to Dr Hank Founds, Thomas Arendt, Reinhard Schinzel and August Heidland for inspirational discussions. Special thanks go to Dörte Fengler, who first noticed the similarity between the structures of thiamine and ALT-711. The authors are not aware of any potential conflicts of interest. 


\section{REFERENCES}

[1] Wolffenbuttel BH, Boulanger CM, Crijns FR, et al. Breakers of advanced glycation end products restore large artery properties in experimental diabetes. Proc Natl Acad Sci USA 1998; 95: 4630-4.

[2] Reiser KM. Nonenzymatic glycation of collagen in aging and diabetes. Proc Soc Exp Biol Med 1998; 218: 23-37.

[3] Candido R, Forbes JM, Thomas MC, et al. A breaker of advanced glycation end products attenuates diabetes-induced myocardial structural changes. Circ Res 2003; 92: 785-92.

[4] Forbes JM, Thallas V, Thomas MC, et al. The breakdown of preexisting advanced glycation end products is associated with reduced renal fibrosis in experimental diabetes. FASEB J 2003; 17: 1762-4.

[5] Jerums G, Panagiotopoulos S, Forbes J, Osicka T, Cooper M. Evolving concepts in advanced glycation, diabetic nephropathy, and diabetic vascular disease. Arch Biochem Biophys 2003; 419: 55-62.

[6] Forbes JM, Yee LT, Thallas V, et al. Advanced glycation end product interventions reduce diabetes-accelerated atherosclerosis. Diabetes 2004; 53: 1813-23.

[7] Holzer H, Kattermann R, Busch D. A thiamine pyrophosphateglycoaldehyde compound ("active glycolaldehyde") as intermediate in the transketolase reaction. Biochem Biophys Res Commun 1962; 7: 167-72.

[8] Akbarian M, Dreyfus PM. Blood transketolase activity in beriberi heart disease. A useful diagnostic index. JAMA 1968; 203: 23-6.

[9] Preuss UW, Soyka M. Wernicke-Korsakow syndrome: clinical aspects, pathophysiology and therapeutic approaches. Fortschr Neurol Psychiatr 1997; 65: 413-20.

[10] Leigh D, McBurney A, McIlwain H. Erythrocyte transketolase activity in the Wernicke-Korsakoff syndrome. Br J Psychiatry 1981; 139: 153-6.

[11] Racker E, De La Haba G, Leder IG. Transketolase-catalyzed utilization of fructose 6-phosphate and its significance in a glucose 6phosphate oxidation cycle. Arch Biochem Biophys 1954; 48: 23840.

[12] Prochoroff NN, Kattermann R, Holzer H. Formation of sedoheptulose-7-phosphate from enzymatically obtained "active glycolic aldehyde" and ribose-5-phosphate with transketolase. Biochem Biophys Res Commun 1962; 9: 477-81.

[13] Cascante M, Centelles JJ, Veech RL, Lee WN, Boros LG. Role of thiamin (vitamin B-1) and transketolase in tumor cell proliferation. Nutr Cancer 2000; 36: 150-4.
[14] Langbein S, Zerilli M, Zur Hausen A, et al. Expression of transketolase TKTL1 predicts colon and urothelial cancer patient survival: warburg effect reinterpreted. Br J Cancer 2006; 94: 578-85.

[15] Thomas AA, Le Huerou Y, De Meese J, et al. Synthesis, in vitro and in vivo activity of thiamine antagonist transketolase inhibitors. Bioorg Med Chem Lett 2008; 18: 2206-10.

[16] Thornalley PJ. Modification of the glyoxalase system in human red blood cells by glucose in vitro. Biochem J 1988; 254: 751-5.

[17] Thornalley PJ, Jahan I, Ng R. Suppression of the accumulation of triosephosphates and increased formation of methylglyoxal in human red blood cells during hyperglycaemia by thiamine in vitro. $\mathrm{J}$ Biochem (Tokyo) 2001; 129: 543-9.

[18] Shangari N, Depeint F, Furrer R, Bruce WR, O'Brien PJ. The effects of partial thiamin deficiency and oxidative stress (i.e., glyoxal and methylglyoxal) on the levels of alpha-oxoaldehyde plasma protein adducts in Fischer 344 rats. FEBS Lett 2005; 579: 5596-602.

[19] Chamberlain BR, Buttery JE, Pannall PR. A stable reagent mixture for the whole blood transketolase assay. Ann Clin Biochem 1996; 33 ( Pt 4): 352-4.

[20] Massod MF, McGuire SL, Werner KR. Analysis of blood transketolase activity. Am J Clin Pathol 1971; 55: 465-70.

[21] Salama G, Lombardi R, Elson J. Maps of optical action potentials and NADH fluorescence in intact working hearts. Am J Physiol 1987; 252: H384-94.

[22] Anderson SH, Nicol AD. A fluorimetric method for measurement of erythrocyte transketolase activity. Ann Clin Biochem 1986; 23(Pt 2): 180-9.

[23] Vasan S, Foiles P, Founds H. Therapeutic potential of breakers of advanced glycation end product-protein crosslinks. Arch Biochem Biophys 2003; 419: 89-96.

[24] Susic D. Cross-link breakers as a new therapeutic approach to cardiovascular disease. Biochem Soc Trans 2007; 35: 853-6.

[25] Little WC, Zile MR, Kitzman DW, Hundley WG, O'Brien TX, Degroof RC. The effect of alagebrium chloride (ALT-711), a novel glucose cross-link breaker, in the treatment of elderly patients with diastolic heart failure. J Card Fail 2005; 11: 191-5.

[26] Food and Nutrition Board IoM. Dietary Reference Intakes: Thiamin, Riboflavin, Niacin, Vitamin B6, Vitamin B12, Pantothenic Acid, Biotin, and Choline, Washington D.C. 1998; pp. 58-86.

(C) Krautwald et al.; Licensee Bentham Open.

This is an open access article licensed under the terms of the Creative Commons Attribution Non-Commercial License (http://creativecommons.org/licenses/by-nc/3.0/) which permits unrestricted, non-commercial use, distribution and reproduction in any medium, provided the work is properly cited. 